Gynäkologe $2011 \cdot 44: 91-92$

DOI 10.1007/s00129-010-2683-5

C) Springer-Verlag 2011

\author{
K. Diedrich ${ }^{1} \cdot$ T. Strowitzki ${ }^{2}$ \\ ${ }^{1}$ UniversitätsklinikumSchleswig-Holstein, Klinik für Frauenheilkunde, Campus Lübeck \\ 2 Universitätsfrauenklinik, Ruprecht-Karls-Universität Heidelberg
}

\title{
Reproduktionsmedizin: Möglichkeiten und Grenzen
}

Sehr geehrte Frau Kollegin, sehr geehrter Herr Kollege,

rechtzeitig zum Erscheinen des Themenheftes über Möglichkeiten und Grenzen der Reproduktionsmedizin wurde im Dezember 2010 der Nobelpreis für Medizin an Herrn Prof. Robert Geoffrey Edwards (- Abb. 1) verliehen. Prof. Edwards hat seit vielen Jahren auf diese Würdigung seines lebenslangen bahnbrechenden Engagements gewartet, die für die gesamte Reproduktionsmedizin eine große Anerkennung bedeutet. Edwards ist Physiologe und war tätig an der Universität Cambridge. Schon in den 1950er-Jahren begann er am Tier intensiv über die Physiologie der Eizelle und die Steuerbarkeit ihrer Reife zu forschen.

In den 196oer-Jahren führte er im Tierexperiment die erste erfolgreiche Untersuchung zur Befruchtung von Eizellen in vitro durch. Trotz intensiver Arbeiten konnte Edwards erst 1978 über die erste Geburt eines Kindes nach In-vitro-Fertilisation unter Embryotransfer berichten: Louis Brown war geboren worden.

Seit dieser Zeit haben sich die Reproduktionsmedizin und die Endokrinologie rasant weiterentwickelt. Das vorliegende Heft gibt einen Überblick über neue Entwicklungen in der Reproduktionsmedizin. Immer mehr in den Fokus des Interesses rückt die nichtinvasive Evaluation des Entwicklungspotenzials von Embryonen.

Es können dafür neue Untersuchungsmethoden der Proteomics und Secretomics eingesetzt werden; diese werden von Germeyer et al. aus Heidelberg beschrieben. Leider können diese Möglichkeiten

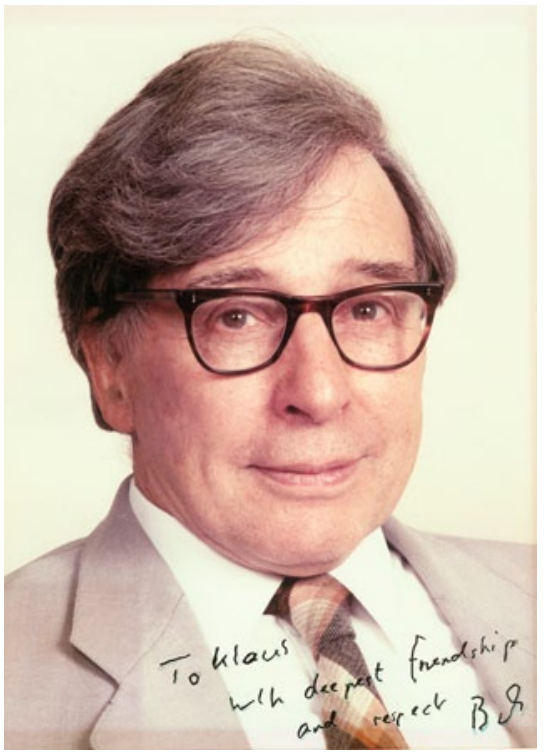

Abb. 1 A Prof. Robert Geoffrey Edwards, Nobelpreis für Medizin 2010

der Embryonenauswahl, die dazu führen, zum einen die Schwangerschaftsrate zu verbessern und zum anderen die Mehrlingsrate zu senken, bisher nur im umliegenden Ausland durchgeführt werden.

Bündgen et al. beschreiben den Stand der Präimplantationsdiagnostik in Deutschland. Sie kann Paaren mit schweren genetischen Erkrankungen die Möglichkeit bieten, ohne eine „Schwangerschaft auf Probe" mit all ihren psychischen und physischen Nebenwirkungen ein gesundes Kind zu bekommen. Das Urteil des Bundesgerichtshofs vom Juli 2010 hat den Weg bereitet für die Behandlung von Paaren mit schweren genetischen Erkrankungen und die Diskussion über ein neues Gesetz zur Regelung der Präimplantationsdiagnostik in Deutsch- land angestoßen. Es wird zu Beginn des Jahres 2011 mit einer gesetzlichen Regelung gerechnet.

\section{(7) Bisher keine verbesserten Schwangerschaftsraten bei älteren Frauen durch PID}

Das Präimplantationsscreening (Aneuploidiescreening) konnte entgegen den Erwartungen bisher keine Verbesserung der Schwangerschaftsraten bei älteren Frauen zeigen. Deshalb kann diese Diagnostik, wie die Polkörperdiagnostik, zum Aneuploidiescreening nicht empfohlen werden.

G. Griesinger nimmt Stellung zu einigen neuen Entwicklungen in der Reproduktionsmedizin, die teilweise kontrovers diskutiert werden. Bevor kostenaufwendige neue Methoden, wie das Assisted Hatching oder die Blastozystenkultur eingesetzt werden, sollten sie zunächst auf den Prüfstein klinischer Studien gestellt werden. Auch wenn Kinderwunschpaare mit wachsender Verzweiflung in zunehmendem Maße bereit sind, experimentelle Methoden in Erwägung zu ziehen - und dafür auch zu bezahlen, so bleibt als ethische Pflicht eine nüchterne Aufklärung über Potenziale und Risiken neuartiger Verfahren, genauso wie das Streben nach Erkenntnisgewinn durch Beteiligung an Forschung und Entwicklung.

In dem Beitrag von Toth et al. werden neue Wege der ovariellen Stimulation und des Single-Embryo-Transfers aufgezeigt. Es wird dafür plädiert, mildere Methoden der ovariellen Stimulation oder auch die In-vitro-Maturation einzusetzen. Diese neuen Verfahren sind prüfenswert, zei- 
gen jedoch derzeit oft niedrigere Schwangerschafts- und Lebendgeburtenraten.

Eindeutig wird für den Single-Embryo-Transfer plädiert, der leider in Deutschland durch die restriktive Gesetzgebung in einem juristischen Graubereich liegt.

Beyer et al. geben einen Überblick über die Vitrifikation von Eizellen im Vorkernstadium und Embryonen. Es zeigt sich, dass die Schwangerschaftsraten nach Vitrifikation etwa denen entsprechen, die auch nach einem Embryotransfer mit frischen Embryonen erreicht werden. Es steht also eine effektive und gute Methoden der Kryokonservierung zur Verfügung.

Wichtig ist immer wieder die Frage, wie es den Kindern nach assistierter Reproduktion geht. Ludwig et al. nehmen hierzu ausführlich Stellung und zeigen das gering erhöhte Risiko, zu früh oder mit einem geringeren Geburtsgewicht geboren zu werden. Es stellt sich jedoch die wichtige Frage, ob diese Ergebnisse am ehesten durch die Subfertilität der Eltern bedingt sein können. Hier müssen sicherlich auch zukünftig weitere Studien gemacht werden.

Mit diesem Themenheft möchten wir Sie mit neuen Entwicklungen in der Reproduktionsmedizin vertraut machen, gleichzeitig jedoch auch Möglichkeiten und kritische Grenzen aufzeigen.

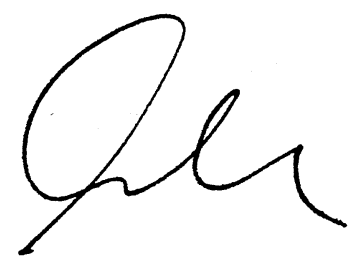

Prof. Dr. Dr. h. c. mult. K. Diedrich
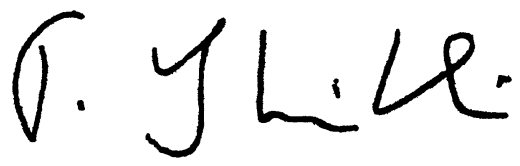

Prof. Dr. Th. Strowitzki

\section{Korrespondenzadresse}

Prof. Dr. Dr. h. c. mult. K. Diedrich UniversitätsklinikumSchleswig-Holstein, Klinik für Frauenheilkunde, Campus Lübeck Ratzeburger Allee 160, 23538 Lübeck klaus.diedrich@uk-sh.de
Der Gynäkologe bietet jeden Monat umfassende und aktuelle Beiträge zu interessanten Themenschwerpunkten aus allen Bereichen der Gynäkologie und Geburtshilfe.

Möchten Sie ein bereits erschienenes Heft nachbestellen? Die bereits erschienenen Ausgaben aus 2010 und 2011 können Sie direkt bei unserem Kundenservice zum Preis von EUR 35,- beziehen:

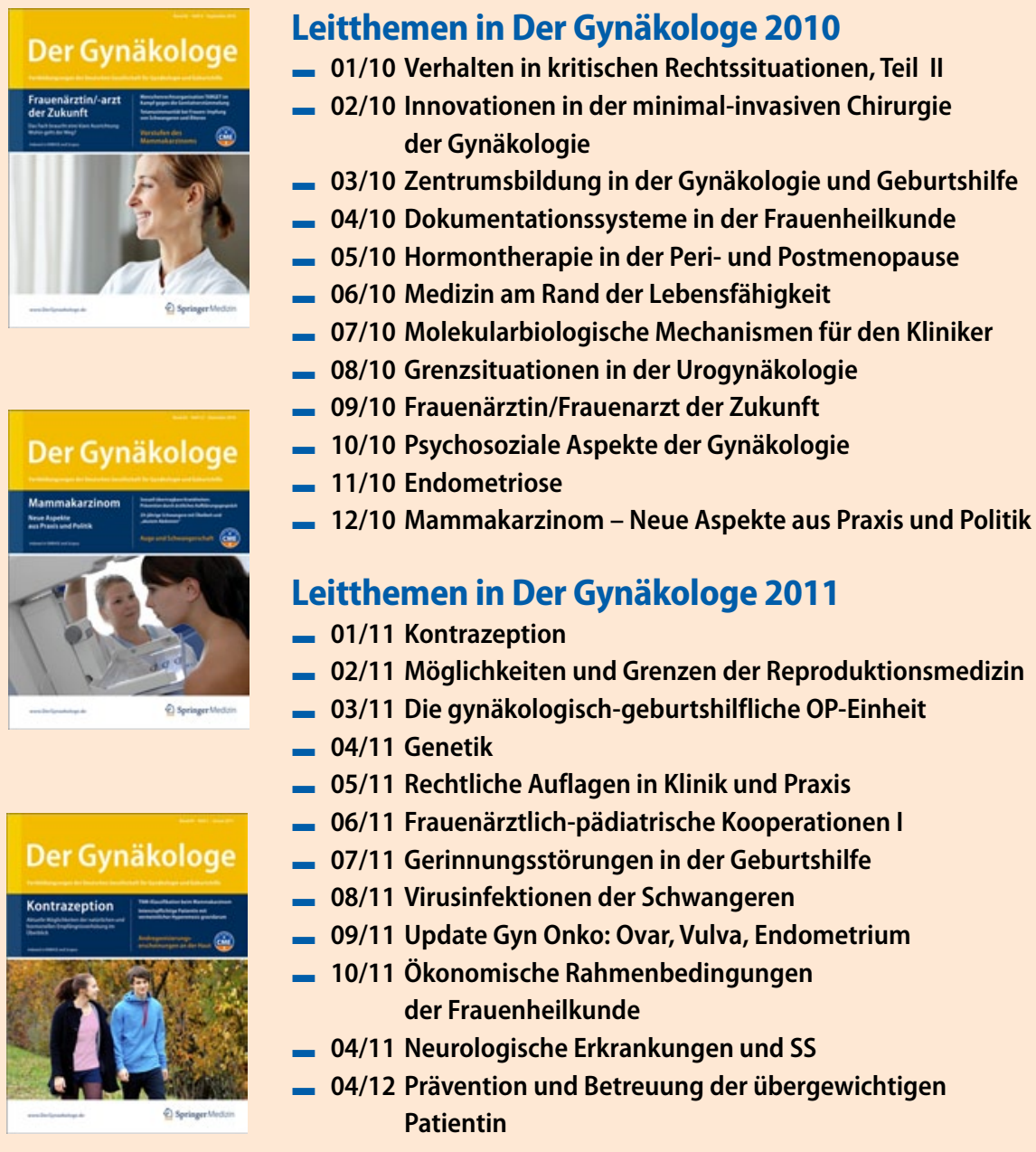

(Änderungen vorbehalten)

So erreichen Sie unseren Kundenservice:

Springer Medizin Verlag GmbH

Kundenservice Zeitschriften

Haberstraße 7

69126 Heidelberg

Tel. +496221 345-4303

Fax: +49 6221 345-4229

E-Mail: Leserservice@springer.com 